La

Révolution

française

\section{La Révolution française}

Cahiers de l'Institut d'histoire de la Révolution française

$16 \mid 2019$

1801-1840 - Haïti, entre Indépendance et

Restauration

\title{
Le projet de Lycée colonial du naturaliste Descourtilz à Saint-Domingue en 1802
}

\section{Vincent Cousseau et Michel C. Kiener}

\section{(2) OpenEdition}

Journals

Édition électronique

URL : http://journals.openedition.org/lrf/2970

DOI : $10.4000 /$ Irf.2970

ISSN : 2105-2557

Éditeur

IHMC - Institut d'histoire moderne et contemporaine (UMR 8066)

Référence électronique

Vincent Cousseau et Michel C. Kiener, «Le projet de Lycée colonial du naturaliste Descourtilz à SaintDomingue en 1802 ", La Révolution française [En ligne], 16 | 2019, mis en ligne le 20 juin 2019, consulté le 28 juin 2019. URL : http://journals.openedition.org//rf/2970 ; DOI : 10.4000/Irf.2970

Ce document a été généré automatiquement le 28 juin 2019

(c) La Révolution française 


\title{
Le projet de Lycée colonial du naturaliste Descourtilz à Saint- Domingue en 1802
}

\author{
Vincent Cousseau et Michel C. Kiener
}

1 Au début de l'année 1802, suite à la cessation des hostilités entre la France et l'Angleterre, la colonie française de Saint-Domingue fait l'objet d'une brutale opération militaire de reconquête voulue par Bonaparte. Elle répond aux intérêts des milieux coloniaux et aux inquiétudes des négociants des ports atlantiques ${ }^{1}$, avec le projet, encore dissimulé, de rétablir l'ordre esclavagiste d'Ancien Régime. Les généraux ne doutent pas du succès, et c'est dans ce contexte de réaction coloniale ${ }^{2}$ qu'ils accueillent le projet de Lycée colonial que leur soumet un jeune médecin naturaliste, Michel Étienne Descourtilz. Celui-ci n'a pas cessé, depuis son arrivée à Saint-Domingue en 1799, d'inventorier le milieu naturel et d'étudier au plus près les populations noires qu'il découvre, dans un monde pourtant en plein bouleversement social et politique.

2 Toussaint Louverture, après avoir bouté les Anglais hors de l'île, actionne tous les leviers pouvant contribuer à consolider son pouvoir personnel. Il rallie avec un certain succès les colons restés dans l'île et permet le retour des émigrés, tout en donnant le change à une France dubitative quant à ses intentions profondes ${ }^{3}$. C'est dans ce contexte que Descourtilz, tout adonné à sa passion naturaliste et à la recherche de protecteurs, propose ses services, disposé à servir le régime légal en place quel qu'il soit. Durant trois années, il accumule des références, missionné successivement par le commissaire français Roume, Toussaint Louverture et Dessalines. Aussi incongru qu'il puisse paraître, ce projet émanant d'un provincial issu d'une famille du Loiret pleinement engagée dans le mouvement révolutionnaire ${ }^{4}$ constitue un bon marqueur des Lumières et d'une science naturaliste en action.

3 Aux archives de la Défense à Vincennes on trouve en effet un curieux document, un « Plan d'organisation d'un Lycé [sic] colonial à établir à St Domingue ", daté du $1^{\mathrm{er}}$ avril 1802, donc rédigé dans la foulée du débarquement de l'expédition du général Leclerc voulue par Bonaparte et censée assurer la reprise d'une colonie en sécession ${ }^{5}$. Les deux feuilles 
manuscrites, adressées "au général Dugas" (en fait Dugua), chef d'état-major de l'expédition, sont signées sur la lettre d'envoi « Descourtilz, médecin naturaliste » et, sur le Plan proposé, « M. E. Descourtilz », un homme dont on ignorait presque tout il y a peu encore.

Un inconnu pour un projet farfelu ? Tout au contraire le témoin précieux d'un continent perdu, le patient travail d'inventaire naturaliste amorcé à la fin du XVIII ${ }^{\mathrm{e}}$ siècle dans la perle des Antilles, au sein même d'une société clivée socialement et racialement. Et cela dans le droit fil du " projet colonial » porté par les institutions scientifiques du royaume de France puis de la République. L'auteur, Michel-Étienne Descourtilz (1777-1838), est bien référencé comme l'auteur d'une Flore pittoresque et médicale des Antilles en huit volumes parue sous la Restauration (de 1821 à 1827$)^{6}$, sans parler des autres travaux portant sur les maladies tropicales, la mycologie et les troubles fonctionnels de la sexualité ${ }^{7}$.

Les recherches menées à l'occasion d'un travail sur les violences à Saint-Domingue l'ont sorti de l'oubli et l'on sait désormais qu'il s'agit d'un médecin naturaliste doué, idéal-type de la nouvelle génération de naturalistes, celle qui doit tout aux Lumières et surtout aux institutions scientifiques et savantes portées par la Révolution ${ }^{8}$. Au retour d'un tragique périple antillais qui faillit lui être fatal, il parvient à faire publier en 1809 ses Voyages d'un naturaliste en trois tomes ${ }^{9}$. Cet ouvrage singulier mêle le récit de ses tribulations et les observations scientifiques qu'il a conduites entre 1799 et 1803 à Saint-Domingue, dans une île alors plongée dans les affres de la guerre civile et coloniale. Les Voyages se présentent donc comme un recueil d'études diverses liées à l'expérience vécue de leur auteur, permettant d'appréhender les conditions de construction du savoir naturaliste dans l'île au plus près du terrain et des acteurs. On suit par là le mode de circulation de l'information scientifique ${ }^{10}$ dans une phase de reconstitution des réseaux de savoir en milieu colonial, aux enjeux certes scientifiques, mais aussi utilitaires et politiques.

\section{Un voyage initiatique, entre science et affaires}

Michel-Étienne Descourtilz, provincial natif d'Orléans, est le fils du régisseur et homme de confiance de l'évêque d'Orléans, acquéreur d'un petit château, puis acteur de la Révolution dans le département du Loiret. Élève au collège d'Orléans puis formé à l'anatomie à l'« école royale de chirurgie » de la ville ${ }^{11}$, comme il le précise lui-même, il monte assez rapidement à Paris poursuivre sa double formation de médecin chirurgien et de botaniste naturaliste, savoirs alors complémentaires. L'anatomie a désormais droit de cité dans l'histoire naturelle avec la dissection systématique de spécimens, en vue d'aboutir aux «principes substantiels permettant d'expliquer un ensemble donné de phénomènes ${ }^{12}$ ». Le jeune Descourtilz semble en effet pénétré, on va le voir, de cette culture nouvelle acquise sous le Directoire, même si l'on peut s'interroger sur les références qu'il invoquera dans sa lettre au général Dugua : «L'étude de la Nature fut dès mon premier âge le principal but de mes occupations. Lié d'intimité avec les Daubenton, Valmont-Bomare, Sage, Charles ${ }^{13}$ etc., je puisai près de ces hommes illustres le germe de connoissances que l'acquit \& l'expérience me font développer de jour en jour ${ }^{14}$. » On ne retrouve pas ici les noms de Jussieu, Lamarck, Desfontaines et André Thouin, qui furent les mentors du médecin naturaliste rochelais Aimé Bonpland (né en 1773), et les figures de proue de l'histoire naturelle de la période révolutionnaire. Quoi qu'il en soit, le jeune Descourtilz se dote lui-même, soit au château familial de Boësses (Gâtinais), soit à Paris, 
d'un « cabinet d'histoire naturelle de phisique et chimie » et, quand il prend la décision de partir pour Saint-Domingue en 1798, il se retrouve, selon ses dires, « chargé par ordre de l'institut National de la description anatomique \& historique du Cayman de St Domingue $»^{15}$.

Il est ainsi porteur d'une culture personnelle doublement moderne : apprenti médecin - il ne passera sa thèse que bien après son retour en France -, il est aussi un Linnéen de naissance pourrait-on dire, formaté par les contacts qu'il a eus à Paris avec les naturalistes travaillant dans les trois ordres de la Nature, minéral, végétal et animal. Son intégration précoce au milieu savant parisien s'accompagne de son entrée en loge. Sa qualité de franc-maçon, qu'il revendique hautement, lui sauve la mise à plusieurs reprises, tant sur mer que sur terre, plongé qu'il est au cœur de l'histoire tragique de Saint-Domingue.

Ainsi formé, et devenu le mari d'une richissime créole de dix-sept ans ${ }^{16}$, le jeune Descourtilz se retrouve missionné par sa belle-famille pour «[re]prendre possession [à Saint-Domingue] de l'habitation de M. Rossignol-Desdunes [...] dont enfin nous venions d'obtenir la levée du séquestre » (Voyages, II, p. 91) ${ }^{17}$. L'enjeu est important puisqu'il s'imagine déjà "propriétaire millionnaire" par sa femme, mais les questions patrimoniales ne l'intéressent guère, et la gestion de domaines moins encore. La mission familiale lui offre surtout l'opportunité d'étudier à peu de frais un monde qui lui est totalement nouveau, avec l'espoir de s'insérer davantage encore dans le milieu savant. De fait, son voyage autofinancé et sa connexion institutionnelle bien ténue font de lui un naturaliste libre de ses mouvements. À ce moment, les autorités françaises ne délivrent des passeports pour Saint-Domingue qu'avec une extrême parcimonie, mais Descourtilz est précisément mandaté par des parents fortunés et propriétaires, qui espèrent recouvrer leurs biens sans en encourir directement les risques. Parti de Paris en mai 1798, il se retrouve toutefois piégé au Havre par le blocus anglais. Il y reste trois mois, pris en mains par des naturalistes de la place qui le promènent et lui enseignent, dit-il, l'art de naturaliser les oiseaux et celui de la peinture au lavis. Finalement, c'est sur un navire américain au départ de Bordeaux, l'Adrastus, qu'il parvient à quitter la France. Après une longue étape à Charleston, il échoue à Santiago de Cuba après avoir subi deux arraisonnements en mer par des croiseurs anglais ${ }^{18}$. Reparti dans une toute petite embarcation avec un couple de parents, il atteint enfin Port-au-Prince le 2 avril 1799.

\section{Un travail en réseau}

Commence alors pour lui une double vie, celle de l'héritier contraint de négocier avec les autorités la restitution effective d'une habitation saccagée du fait des troubles et d'une hatte (haras) à l'abandon - dites de l'Écluse et situées dans l'Artibonite -, et celle d'un " amateur passionné » (comme il se qualifie lui-même) qui se jette à corps perdu dans l'histoire naturelle du pays. À peine arrivé, il est reçu à bras ouverts par le secrétaire du commandant de la place de Port-au-Prince, botaniste distingué qui possède en propre un jardin des plantes. Et il va très vite trouver, au Cap, des familiers du pays qui seront ses initiateurs et qui forment entre eux comme une société de botanistes, plus ou moins héritiers du Cercle des Philadelphes formé en 1784 et dissout en 1793. Roume, le nouvel agent du gouvernement français nommé à Saint-Domingue après le départ d'Hédouville, partage leur passion ${ }^{19}$. Il faut dire que les marges d'action de Roume sont très limitées, le pouvoir effectif étant concentré entre les mains de Toussaint Louverture, devenu le 
véritable maitre de lî̂le et qui ne souffre aucune concurrence. Ravi de la présence d'un jeune naturaliste enthousiaste, Roume décide aussitôt de le missionner (Voyages, III, p. 14) pour qu'il entreprenne le travail sur le caïman « qu'on demandait de France » (Voyages, III, p. 241) et poursuive l'inventaire botanique de l'île.

Dès lors, Descourtilz, bon cavalier, ne va plus cesser de parcourir le pays. "J'aimais à Saint-Domingue, à observer les beautés de la nature [...], je fuyais les hommes... », c'est-àdire les mondanités, déclare-t-il en promeneur solitaire rousseauiste (Voyages, II, p. 383), mais il n'en met pas moins au jour pour nous tout un réseau d'hommes de savoir dont il devient, vu sa jeunesse, un élève ou, au mieux, un partenaire de travail. Car, si la nature sauvage le fascine et s'il s'intéresse au comportement animal, il est moins un contemplateur qu'un homme de prélèvement. Ainsi, il s'adonne en permanence à la chasse et relève, sous cet angle, de la figure du "savant chasseur" réprouvée par Bernardin de Saint-Pierre. Le jeune Descourtilz est bien un observateur méthodique et infatigable, mais sa démarche ne s'inscrit pas dans le principe de restauration d'une harmonie naturelle et antéhistorique avec les animaux ${ }^{20}$. En janvier 1800, il tombe à l'habitation Robuste, près du bourg de Petite-Rivière, sur François-Richard de Tussac, colon lui-même, qui travaille depuis dix ans à une Flore des Antilles qui sera publiée en France en $1808^{21}$ (Voyages, II, p. 405). Quelques jours après, Tussac, chez qui il fait étape à Saint-Marc, lui ouvre ses boîtes et lui montre ses planches dessinées par un autre, tandis qu'arrive à cheval un certain Chatainier, chargé à son tour de planches de dessins, et tous trois repartent de concert en herborisant sur le chemin du Cap (Voyages, II, p. 406). Le 17 janvier 1800, il rend visite au général Moyse, neveu de Toussaint Louverture qui commande la partie nord de l'île et qui se montre intéressé par les travaux des naturalistes. Il parcourt en compagnie de Tussac le jardin botanique de l'hôpital alors que la révolte gronde (Voyages, II, p. 412-413), puis revoit Roume, lui-même marié à une créole du pays, qui lui fait voir « son laboratoire de chimie et sa collection d'Histoire naturelle » (Voyages, II, p. 414). Il rencontre en outre, dit-il, trois autres naturalistes ${ }^{22}$, puis Luziès, un pharmacien, et Daubertès, chirurgien en chef, qui ont tous deux des collections, Daubertès s'étant focalisé sur les coquillages.

11 La guerre civile et les suites de l'expédition Leclerc à Saint-Domingue peuvent faire paraitre dérisoire à nos yeux le travail de ces naturalistes, obstinés en dépit de tout à poursuivre le travail d'inventaire amorcé sous l'Ancien Régime, démontrant par-là la force de séduction du travail de l'intelligence du monde, qu'il s'inscrive consciemment ou non dans un «projet colonial $»^{23}$. Dès 1790, Saint-Domingue comptait l'un des premiers et rares correspondants de la toute nouvelle Société d'histoire naturelle de Paris (de Genton, présenté par Faujas ${ }^{24}$ ) et, dans le cours de la décennie, les naturalistes du cru n'ont cessé d'accueillir de nouveaux acteurs, parmi lesquels le jeune Hyppolite Nectoux ${ }^{25}$. Celui-ci, arrivé à Port-au-Prince en janvier 1788, est rapidement nommé directeur du Jardin botanique du roi à Saint-Domingue ${ }^{26}$ et multiplie les travaux huit ans durant, jusqu'à ce que les troubles en 1796 l'obligent à fuir Saint-Domingue pour se réfugier aux États-Unis. Il confie ses apports aux botanistes de l'île : l'arbre à pain de Tahiti, et des girofliers et des canneliers pris à Cayenne - des plantes que Descourtilz retrouvera en 1799 sur une habitation (Voyages, II, p. 325). Dans le même temps, deux hommes composent un improbable duo dans ce Saint-Domingue secoué par les révoltes : un jardinier bien connu du Jardin des plantes parisien, Antoine Poiteau (1766-1864), et le sergent Jean-François Turpin (1775-1840), dessinateur de talent. Poiteau collecte et décrit, Turpin dessine, et tous deux ne repartent de Saint-Domingue qu'à l'extrême fin de la décennie 1790, au 
moment où arrive Descourtilz. Voici enfin, en 1797-1798, un ingénieur sorti de l'École des mines, Alexandre-Philippe Advenier, minéralogiste du premier voyage du capitaine Nicolas Baudin, chargé par le Directoire en 1796 d'une première mission dans la zone caraïbe, et qui rapportera en France des «centaines de caisses » en juin $1798^{27}$. Baudin, resté à Porto Rico, envoie Advenier dans l'île, où il passe neuf mois à dresser un premier inventaire des richesses minières de la partie espagnole. En fait, celui-ci reste sur place, puis collabore avec Descourtilz, qui le fait figurer en 1802 dans la liste des hommes partie prenante de son projet de lycée : il est à cette date, dit Descourtilz, l'un des « naturalistes actuellement dans la Colonie », parmi les quatre alors présents au Port-au-Prince et les trois au Cap ${ }^{28}$.

De tout cela, des travaux d'Hypolite Nectoux et de tant d'autres il ne reste hélas rien ou presque, sinon de rares archives, du fait des incendies et des saccages liés aux troubles, des naufrages (fréquents) et des saisies par les corsaires et les croiseurs anglais. La plus grande partie des travaux de Descourtilz seront abandonnés sur place ou partiront en fumée, tout comme deux mille dessins de François-Richard de Tussac. Quant à Turpin et Poiteau, ce sont leurs travaux de France et d'après le tournant du siècle qui feront seuls leur réputation, quand bien même ils auront auparavant arpenté Saint-Domingue en tous sens.

En attendant, c'est avec ce riche capital de contacts et d'informations disponibles que le jeune Descourtilz, tout juste vingt-deux ans, va entamer son inventaire personnel de l'île. Et c'est ce même environnement scientifique qui va lui permettre d'imaginer et de proposer aux autorités en 1802 un travail d'équipe à mener dans un Lycée susceptible d'asseoir les lumières de la science dans un pays en plein bouleversement social et politique.

\section{Descourtilz tous azimuts}

14 À peine arrivé en avril 1799, Descourtilz se lance dans l'étude du crocodile (ou caïman) de Saint-Domingue. Il mène son affaire rondement et dissèque minutieusement, en quelques mois, cinquante-sept sujets de toutes tailles et de tous âges, ce qui lui permet de confronter ses trouvailles - notamment le squelette - aux dires de Geoffroy Saint-Hilaire (qu'il valide), et de Lacépède (qu'il conteste) (Voyages, III, p. 17-24). Il multiplie les planches de détail, il étudie les mœurs et le comportement de l'animal, tirant pour finir de ses observations une Histoire naturelle du crocodile de Saint-Domingue appelé Caïman en huit chapitres, un "ouvrage compliqué » dont Roume, qui l'a «pris sous ses ailes protectrices » lui a « facilité la conception » (Voyages, III, p. 13). Ce travail sera présenté à l'Institut à son retour en France et fera l'objet en 1807 d'un compte rendu détaillé signé Tenon et Lacépède, et Cuvier rapporteur ${ }^{29}$.

Il travaille avec d'autant plus d'acharnement qu'il pense rester peu de temps dans l'île ( Voyages, II, p. 90). Il tient un journal de ses recherches (Voyages, II, p. 451), observe tout ce qu'il peut dans les trois ordres de la nature et entreprend pour son compte au cours de cette année 1799 un inventaire rapide de la faune et de la flore du pays, en commençant par les mammifères - cabris, bovins et porcs « marrons » - ; puis il s'intéresse aux reptiles - tortues et caïmans -, aux oiseaux - ramiers, tourterelles... -, il collectionne les plantes et retrouve "le dimanche 2 juin 1799 ", durant une visite à l'habitation T*** (trois points disposés en triangle), canneliers, girofliers et muscadiers, dont on a vu l'introduction toute récente par Hypolite Nectoux. Des bambous (Voyages, II, p. 325), il passe aux 
scorpions... Arpentant le territoire, il découvre le monde des travailleurs noirs et de leurs pratiques secrètes, et se lance dès lors dans l'étude des «mœurs des Africains » (Voyages, III, p. 112) et des différentes ethnies, qu'il identifie au plus près du terrain. Moreau de Saint-Méry venait de livrer au public des éléments sur les esclaves arrivés d'Afrique selon la perspective géographique, physique et morale propre aux planteurs ${ }^{30}$. Descourtilz livre en revanche une enquête ethnologique et anthropologique avant la lettre, qui n'est pas sans rappeler les préoccupations des promoteurs de la Société des observateurs de l'homme fondée en $1800^{31}$. Une bonne maitrise des parlers créoles vite appris - il est un musicien confirmé - va l'aider dans son entreprise. Il en tire une très longue dissertation en quinze chapitres qui fait suite dans le troisième volume des Voyages à l'étude sur le caïman : l'Essai sur les mœurs et coutumes des habitans de Guinée, à Saint-Domingue, pour servir à l'Histoire générale de l'Afrique ${ }^{32}$. Sa démarche comme ses conclusions sont à rebours de celles de Julien-Joseph Virey ${ }^{33}$, la disparité des mœurs observables se détachant d'une interprétation environnementaliste et ne débouchant ni sur la classification émergente en " races ", ni sur une hiérarchisation des nations observées.

Début février 1800, il est missionné à nouveau par Roume pour poursuivre l'exploration des mines abandonnées de la partie espagnole commencée par Advenier, ce qui nous vaut de riches tableaux pliés et insérés dans le tome II des Voyages (427-434). Ayant fini ses "courses d'Histoire naturelle» et étant bloqué dans ses démarches administratives concernant les biens familiaux, il accepte une place qui lui est offerte à bord d'une frégate qui doit rallier la France. Mais Toussaint Louverture le retient en le faisant appeler à son état-major du Cap. Il avait déjà eu affaire à lui dès son arrivée, Descourtilz lui ayant remis en main propre les lettres de ses fils scolarisés à Paris, et s'étant entretenu avec lui pour des questions privées (Voyages, II, p. 71-72). Désireux de le conserver à Saint-Domingue, Toussaint lui offre un sauf-conduit, daté du 20 floréal an VIII (10 mai 1800), pour lui permettre de parcourir librement toute l'île, «l'objet de ses recherches étant de faire florir les sciences » (Voyages, II, p. 447-448). Dès lors, le voici personnage officiel auquel nul ne saurait toucher parmi les subordonnés de Toussaint Louverture, ce qui lui vaut la précieuse protection du général Dessalines de passage sur l'habitation familiale ; celui-ci avertit les cultivateurs de bien prendre garde à ce qu'il ne lui arrive rien. Descourtilz représente non seulement la caste des propriétaires blancs éclairés, à ce moment préservés par Toussaint Louverture, mais surtout l'une des compétences scientifiques nécessaires à l'édification d'un nouvel État.

En 1799-1800, Descourtilz s'est donc employé à mériter la confiance que l'administrateur Roume et le maitre noir du pays lui ont faite. Il ne se contente pas de récolter, il rédige, dans l'intention de publier. Ultérieurement, il expliquera que, une fois « l'ouvrage achevé [son traité sur le caïman ${ }^{34}$, je me livrai à un traité des plantes usuelles de $\mathrm{S}^{\mathrm{t}}$. Domingue, $\mathrm{Gd}$ in folio, orné de planches enluminées, puis successivement à mon grand ouvrage du Contemplateur ${ }^{35}$. » Il se fait même connaître des praticiens de l'île par " [ses] ouvrages sur la médecine » (Voyages, III, p. 303). Sans en être conscient - à moins qu'il s'en soit nourri -, Descourtilz a ainsi comblé les vœux de Volney tels qu'il les exprime dans ses Questions de statistique à l'usage des voyageurs parues en 1795 , en n'attaquant le travail de synthèse qu'après s'être donné une solide connaissance personnelle du terrain ${ }^{36}$. "Pour bien questionner, insiste Volney, il faut avoir déjà une idée des objets vers lesquels tendent les questions ». Pour passer - disent les instructions données en 1795 par l'administration à ses agents et citées par Volney - de la « curiosité » à « l'instruction » (à la connaissance) des réalités d'un pays, pour «la science si importante de l'économie publique », il faut 
accumuler les observations sur les rapports et les contrastes « dans le climat, le sol, les produits naturels et toutes les circonstances physiques et morales $»^{37}$.

Mais l'histoire se précipite, avec l'expédition du général Leclerc voulue par Bonaparte et qui arrive en février $1802^{38}$. Toussaint Louverture, submergé par l'attaque, impuissant à entrainer la population dans la résistance et abandonné par ses généraux, se voit contraint de rendre les armes ${ }^{39}$. Pour Descourtilz comme pour tant d'autres, le cours de l'existence dérape : arrêté et pris en otage par les insurgés, il vit en février et mars, en témoin tétanisé, des semaines tragiques, sans cesse menacé par l'exécution à laquelle il réchappe à plusieurs reprises grâce à ses connaissances médicales. Il parvient à s'enfuir ${ }^{40}$ et, avant que se déchainent sur place les factions et les rivalités, il profite d'une courte fenêtre de tir, en avril 1802, pour présenter aux autorités françaises un projet qui lui tient à cœur.

\section{Le projet de lycée colonial}

En avril 1802, oublieux des réalités humaines, raciales et politiques du moment, et dans un contexte militaire encore incertain, Descourtilz pense pouvoir mobiliser les intelligences en vue de poursuivre l'œuvre d'inventaire qu'il a commencée. C'est ce qu'implique le Plan d'organisation d'un lycée colonial à établir à Saint Domingue, [daté en marge] $1^{\text {er }}$ Avril 1802/11 Germinal An X, un projet très probablement mûri de longue date qu'il remet au général Dugua, chef d'état-major général de l'armée. Celui-ci - explique Descourtilz - avait, quelques jours avant, « examiné mes manuscrits restés en dépôt en cette ville, et les seuls que j'avois échappés aux flammes ». Résilience hors normes d'un jeune homme qui, tout juste sorti des scènes tragiques dont il a été témoin, adresse sans délai aux « lumières » du général « le premier effort d'un génie comprimé par d'affreux revers ». Mais c'est aussi cette hâte à produire un texte qui explique le caractère sommaire de l'exposé, réduit à une lettre recto verso et à une feuille en forme de tableau. Le projet donne corps au réseau informel qu'il s'est créé en trois années, à un moment où la colonie n'a plus ni société savante ni même lieux d'échanges appropriés. Il s'agit donc de combler un vide institutionnel et de lancer un véritable programme de recherche structuré et durable.

Est-ce bien le général Dugua qui a «proposé d'établir, par sa Correspondance alternative du Cap au Port-au-Prince » une "Société » qui " ne négligera rien pour faire tourner ses recherches au profit du Gouvernement français et [...] diversifiera ses expériences en portant de tems à autre ses observations vers les divers points de l'histoire naturelle, phisique chimie \& anatomie «? Descourtilz, dans le style de l'époque, en laisse l'initiative au général, mais il n'en dicte pas moins ses conditions. On ne peut s'empêcher de penser aux projets de Pierre-Joseph Briot (1771-1827), qui propose aux alentours de 1798-99 de créer cinq lycées, à Bruxelles, Dijon, Toulouse, Paris et Poitiers, institutions intermédiaires entre l'Institut et les écoles centrales ${ }^{41}$. Descourtilz, à l'évidence, entend dépasser le travail mené jusque-là au sein de son cercle d'amis. Ce Plan d'organisation d'un lycée colonial doit conduire à la mise en place d'un personnel dédié à un projet collectif, avec des membres «reconnus et nommés par les autorités compétentes ", munis d'un "brevet » et vêtus d'un " costume [...] propre à inspirer la confiance ", comme en ont les personnels des « établissements de ce genre » en France; ils pourront alors «poursuivre leurs recherches sans être troublés par quoi que ce soit [...] avec sûreté et recommandation auprès des autorités civiles et militaires. » 
Mais Lycée qu'est-ce à dire ? C'est travailler dans l'esprit du Lycée péripatéticien fondé à Athènes par Aristote, qui assignait des missions de recherche précises à ses membres et ses élèves au service de la connaissance. Descourtilz fournit donc au général un Exposé des questions à résoudre par le Lycé colonial de St Domingue sur divers points de l'histoire naturelle de ce pays appliquables à l'utilité publique. De ces "questions", il dresse une première liste, rangées dans les trois colonnes d'un tableau manuscrit, transcrit ci-dessous, consacrées aux trois règnes de la Nature :

- Minéral : avec des questions sur l'air, l'eau, les terres, la pierre et les mines.

- Végétal : l'importance des plantes et des fruits pour les hommes et l'industrie.

- Animal: dans une classification par disciplines autonomes, celle qui s'impose dans les années 1790 et ouvre le champ à la professionnalisation savante ${ }^{42}$.

Exposé de questions à résoudre par le Lycé Colonial de St Domingue sur divers points de l'histoire Naturelle de ce pays appliquables à l'utilité publique,

Présenté au Général Dugas par M. E. Descourtilz

\section{Règne minéral}

L'air. De son influence maligne au commencement des plaies, produite par la décomposition des substances qui entrent en fermentation. Remèdes à opposer à ses funestes effets. Causes des fièvres d'alors.

L'eau. De leur analyse, et de leur influence au mois de may, juin etc. sur la santé. Des eaux minérales.

Terres et sables. Les plus utiles \& les plus propres aux besoins de l'homme, dans l'édification des batiments. Examen des terres salines produites par divers cantons, contenant nitre, soude, natron, vitriol etc. sel gemme.

Pierres. De celles les plus propres à la bâtisse, de la position desdites carrières albâtres et cristaux des grottes.

Souffres et bitumes. De l'exploitation des souffrières des montagnes de la Selle et autre grottes, mornes à volcans. Grands Cahos etc.

Mines. De la visite des mines les plus dignes d'être exploitées.

\section{Règne végétal}

$1^{\circ}$ Plantes médecinales appliquables à l'économie de l'homme et des animaux.

$2^{\circ}$ Plantes dont les sucs concrets ou fluides deviennent utiles aux arts.

$3^{\circ}$ De l'influence des vivres ou fruits sur le tempérament des Créols, \& des Européens, non acclimatés.

$4^{\circ}$ Sur les ressources pour les arts renfermés dans une infinité de plantes, tels que celles à coton dont on fait des chapeaux. Les pitt et écorces souples pour les fins \& gros cordages.

$5^{\circ}$ Sur les gommes et brais déposés par la Nature dans les riches arbustes.

$6^{\circ}$ Bois de construction.

$7^{\circ}$ De l'antidote à trouver pour la neutralisation des poisons du pays.

$8^{\circ}$ D'un traitement curatif des maladies vénériennes par les plantes du pays. 


\section{Règne animal}

Histoire des quadrupèdes. L'agouti qui se trouve fort rarement dans les montagnes du gros morne, et dans la partie du Sud est le seul quadrupède indigène. Remèdes idoines à opposer à l'épizootie des animaux de Hatte \& autres.

Ornithologie. Il est des oiseaux dont le plumage est recherché dans les parures et devient précieux aux modistes, et plumassiers. Avantages qu'on retireroit de la domesticité de plusieurs gibiers de St Domingue.

Ictyologie. Il sera bon de s'occuper de la connoissance des poissons communs aux parages; de ceux dangereux à manger, des époques où leur chair est funeste; de la propriété de leurs huiles relativement à la peinture etc.

Entomologie. Cette classe fournit plusieurs mouches épipastiques, appliquables à la pharmacie. Une lachenille qui se trouve dans des cardasses de l'Artibonite. Moyens de prévenir les suites des morsures insectes venimeux.

Conchyologie. Cette classe fournit de très riches sujets.

Zoophytes. On peut à St Domingue en recueillir une complètte collection.

Reptiles et serpents. Leur graisse élaborée devient utile à certaines maladies.

\section{Nota}

Noms des naturalistes actuellement dans la Colonie

Au Cap : Mrs Tussac, L'abbé de la Haie, Daubatie chirurgien

Au Port-auprince : Mrs Descourtilz, Haugard, Advénier, Dabeneaud

Dans l'optique de Descourtilz, la colonie n'est plus un simple lieu de collecte ou même d'observation, mais un centre autonome de production du savoir. L'air de rien, le jeune naturaliste propose une forme de décentralisation scientifique, à contre-courant de l'évolution récente. En effet, le Muséum d'histoire naturelle affirme alors sa vocation à centraliser les collections naturalistes en s'appuyant sur un réseau de voyageurscollecteurs, principalement animé et coordonné par la Société d'histoire naturelle de Paris ${ }^{43}$. En promouvant un développement exogène de la recherche, le Plan de Descourtilz apparaît donc en décalage avec le tropisme triomphant de l'organisation centralisée de la construction des savoirs ${ }^{44}$. Sa proposition répond moins à un objectif politique assumé qu'à une démarche scientifique alternative, qui transparaît dans ses Voyages, notamment dans ses pages détaillées consacrées au caïman. En effet, Descourtilz privilégie une science fondée sur l'observation directe des végétaux et des animaux dans leur milieu, à rebours de l'acclimatation dans des jardins botaniques ou de l'étude en cabinet de spécimens préparés. Cette démarche possède non seulement d'évidents avantages pratiques, mais elle permet surtout l'étude des différentes espèces dans leur écosystème, l'observation de leur adaptation et, pour les animaux, de leurs comportements. Novateur sur le plan institutionnel, le Plan d'organisation propose en outre une démarche scientifique alternative, fondée sur une observation au plus près du vivant. 


\section{En quête de l'utile}

milieu. Les « questions à résoudre » listées par Descourtilz dans son manuscrit sont toutes
pratiques et destinées à servir les « arts », c'est-à-dire l'industrie des hommes, «l'étude de l'histoire naturelle devant préférablement avoir pour but les avantages qu'on peut retirer des productions terrestres ou aquatiques». Les plantes? Rechercher celles qui sont «appliquables à l'Economie de l'homme et des animaux» et celles «dont les sucs concrets ou fluides deviennent utiles aux arts", et puis le coton "dont on fait des chapeaux", les écorces souples "pour les fins \& gros cordages». Ayant lui-même échappé de peu à un empoisonnement, fléau hérité de la société esclavagiste, il n'oublie pas la recherche de l'antidote pour les neutraliser et, plus largement, les plantes médicinales. Les terres? « Les plus utiles et les plus propres aux besoins de l'homme, dans l'édification des bâtiments ", les terres salines pour la soude, le vitriol etc. Et puis le souffre, les bitumes et les mines « les plus dignes d'être exploitées. » Quant aux animaux, il en va de même : étudier les épizooties, rechercher les oiseaux «dont le plumage est recherché dans les parures" par les modistes, identifier les poissons "dangereux à manger ", étudier les propriétés des huiles « propres à la peinture ", etc. Il s'agit d'abord de proposer une liste de produits qui relèvent davantage du prélèvement ou de la prédation que du développement de l'agriculture ou de l'élevage.

Cette soudaine obsession de l'utilité publique pose question quand on se rapporte aux travaux effectifs de Descourtilz, « médecin naturaliste » avant tout soucieux de découvrir et de décrire, et qui continue à l'hiver 1802-1803 d'herboriser et de collecter à marée basse les coquillages qu'il souhaite dessiner (Voyages, III, p. 379). En 1795, le questionnaire Volney, où étaient détaillés les points à travailler lors des expéditions scientifiques, n'avait pas ce caractère si étroitement utilitaire ${ }^{45}$. Un an plus tard, en 1796 , les instructions données par le Directoire au capitaine Nicolas Baudin, auquel on confiait une flûte, La Belle Angélique, allaient dans le même sens : l'expédition n'avait de chances de réussite dans l'univers si complexe des Antilles partagé entre nations concurrentes ou ennemies, qu'en conservant un caractère exclusivement scientifique et en s'interdisant tout commerce ${ }^{46}$. En 1818 encore, l'Instruction pour les voyageurs et pour les employés dans les colonies... par l'administration du Muséum royal d'histoire naturelle ${ }^{47}$ conserve le caractère d'un programme de recherche exclusivement scientifique, en se limitant aux instructions et aux conseils liés à « la recherche, la préparation et l'envoi » de spécimens recueillis lors des missions savantes. Pour autant, on est aussi dans cette période où la science ouverte sur l'homme, la nature et les animaux évolue en une science du classement et de l'ordonnancement utilitaire ${ }^{48}$. Depuis les débuts de la Révolution, la question de l'utilité publique de la science s'est posée, en particulier à propos du devenir du Jardin du Roi, avec la volonté de dépasser l'activité élitiste de loisir savant et de penser la science comme un levier de la Régénération. Sa transformation en juin 1793 en Muséum d'histoire naturelle suscite une réflexion inédite sur la portée politique de la science et l'articulation entre l'homme et les animaux ${ }^{49}$. Ainsi la question de l'utilité économique du projet de ménagerie, négligée dans un premier temps par Bernardin de Saint-Pierre, est habilement réintroduite par la Société d'histoire naturelle, permettant d'emporter la conviction de la bourgeoisie parlementaire. Avec le député Thibaudeau, son successeur thermidorien et conservateur, l'utilitarisme se renforce en prenant un tour patriotique : 
assurer l'indépendance de la France ${ }^{50}$. Au sein de la Société d'histoire naturelle, AubinLouis Millin souligne la nécessaire recherche du «but utile ", proscrivant une utilisation de l'argent du peuple à seule fin de "satisfaire une vaine curiosité ", mais sans pour autant céder à un utilitarisme à courte vue et renoncer à la liberté académique ${ }^{51}$. En juillet-août 1793, cette Société, consacrant plusieurs séances à "discuter le projet d'instruction publique de l'histoire naturelle " à présenter à la Convention, pose le principe de la « Nécessité de l'histoire naturelle pour enrichir la République, préliminaire nécessaire des arts utiles » (31 juillet). Ce lien entre l'inventaire de la planète, les « arts » et l'utilité publique est au cœur de la longue Adresse à la Convention dont «le citoyen Hallé » soumet le texte à ses pairs le 9 août. Celui-ci rappelle que l'homme, dès l'origine "placé au milieu du théâtre de l'univers », n'a progressé qu'en trouvant « les substances propres à le nourrir », les terres « qui donnent à ses vases le plus de solidité ", etc. ; « c'est toujours pour les arts que l'homme instruit, en observant la nature, l'agrandit, la féconde et double ses richesses. Combien de fois le naturaliste voyageur a-t-il enrichi les manufactures et le commerce de productions étrangères [...], l'observateur a-t-il montré dans l'arbre ou la plante indigènes un supplément économique ", découvrant "des végétaux utiles qui croissaient et périssaient inutilement pour les arts $»^{52}$ ? Quant aux colonies elles-mêmes, ajoute Hallé, «si quelque genre d'instruction peut être avantageux à la prospérité nationale dans les colonies, c'est certainement celui qui concerne l'histoire naturelle ${ }^{53} »$.

De fait, le lien entre histoire naturelle et les «arts » revient sans cesse désormais, de façon implicite ou explicite, dans les écrits et les justifications des naturalistes voyageurs ${ }^{54}$. La Nature peut et doit contribuer au développement de la civilisation et au bonheur. Dans cette perspective, le voyage naturaliste s'institue en geste "d'appropriation intellectuelle et physique du monde qui mélange utopie, science, profit personnel, utilité publique et esprit de conquête ${ }^{55}$ ».

26 Le Projet de Descourtilz semble imprégné de cette atmosphère. Son tour très économique a certainement été renforcé par l'intervention des généraux de l'expédition Leclerc, anciens de l'expédition d'Égypte, Leclerc lui-même, Dugua et Thouvenot, qui ont pour mission la reconquête d'un territoire à forte valeur économique, dans un aggiornamento de l'esprit scientifique et pratique de la « Machine coloniale » qui caractérisait les ambitions de la monarchie ${ }^{56}$. Dugua, au nom de «l'intérêt que le Premier Consul [prend] aux beaux arts », nomme Descourtilz « médecin-naturaliste du Gouvernement » avec un traitement annuel de 6000 francs; il lui remet "au nom du général Leclerc, la décoration particulière d'une ceinture noire, ou cordon de mérite, comme fondateur du lycée colonial », ce qui le « mettoit dans le cas d'avoir l'honneur de correspondre avec l'Institut national » et d'en devenir « membre honoraire » (Voyages, III, p. 375). De son côté, Leclerc, qui pousse à la reconstitution d'une société savante au Cap, réclame à Chaptal l'envoi de six botanistes de France $^{57}$. Pour ces généraux, même en pleine phase d'opérations militaires et de pacification, la science reste un enjeu politique et symbolique. À un moment où le Premier Consul se montre parcimonieux dans l'envoi de renforts militaires, les chefs militaires de l'expédition doivent montrer que la vie dans la colonie peut reprendre dans tous ses aspects et donner des gages sur son utilité économique, alors bien amoindrie.

Quelques mois plus tard, le général Thouvenot, « juste appréciateur des sciences que luimême cultive ", met à la disposition de Descourtilz un local situé à l'état-major pour y travailler à la mise au net de ses manuscrits. En vain. Ce lycée demeura comme le reste à 
l'état de projet. Tandis que les dernières troupes françaises se repliaient sur quelques points de la côte, "plusieurs officiers généraux m'engagèrent à sauver mes manuscrits ", nous dit Descourtilz, qui est littéralement exfiltré en se voyant attribuer en mai 1803 par le capitaine-général Rochambeau une place privilégiée à bord d'une corvette rapide à destination de l'Espagne. Sauvant ainsi d'une saisie anglaise une partie du travail d'inventaire réalisé dans un empire colonial en perdition, conservé tel un maillon pour de futures études ${ }^{58}$.

\section{NOTES}

1. Manuel covo, Commerce, empire et révolutions dans le monde atlantique. La colonie française de SaintDomingue entre métropole et États-Unis (ca. 1778 - ca. 1804), thèse de doctorat d'histoire, sous la direction de François Weil, 2013, p. 720-721, 733-739.

2. Voir à ce propos Yves BENOT, La démence coloniale sous Napoléon, Paris, La Découverte, 1992.

3. Laurent DUBoIs, Les Vengeurs du Nouveau Monde. Histoire de la Révolution haïtienne, Rennes, Perséides, 2005, p. 307-308.

4. En novembre 1793, le père de Michel Étienne et son gendre sont tous deux administrateurs du Loiret (Arch. dép. Loiret, 617 O-Suppl 1 E/2, vues 15-16).

5. Service historique de la Défense, GR B7 13.

6. Michel-Étienne DESCOURTILZ, Flore pittoresque et médicale des Antilles ou Histoire naturelle des plantes usuelles des colonies françaises, anglaises, espagnoles et portugaises... peinte d'après les dessins faits sur les lieux par M. J. Th. Descourtilz [son fils], Paris, Pichard, 1821-1827, 8 vol.

7. Après sa thèse de médecine, soutenue seulement en 1814 , il est brièvement directeur de l'hospice de Beaumont-en-Gâtinais (Seine-et-Marne), avant de s'installer définitivement à Paris pour retrouver le milieu des naturalistes et la Société Linéenne, dont il est, un temps, le secrétaire. Il publiera le Guide sanitaire des voyageurs aux colonies, ou Conseils hygiéniques en faveur des Européens destinés à passer aux îles..., Paris, C.-L.-F. Panckoucke, 1816; Des Champignons comestibles, suspects et vénéneux... par M.-É. Descourtilz, Paris, Chappron, 1827 ; De l'Impuissance et de la stérilité, ou Recherches sur l'anaphrodisie distinguée de l'agénésie. Ouvrage destiné aux Personnes mariées qui ne peuvent avoir d'enfants, Paris, Masson et Yonet, 1831, 2 vol.

8. Voir sa biographie dans Vincent COUSSEAU \& Michel C. KIENER, La Révolution à Saint-Domingue : Récits de rescapés (1789-1804) J. M. Bonjour, M. É. Descourtilz, J. Decout, 35-Bécherel, Éditions Les Perséides, 2016, p. 75-87. Il faut y ajouter les informations tirées du fonds $293 \mathrm{~J} 4$ des Archives départementales de la Seine-et-Marne concernant sa vie après son retour en France en 1803 et sa belle-famille créole.

9. Paris, Dufart père, 1809.

10. Cf. à ce propos la thèse de Thérèse $\mathrm{BRU}$, Les naturalistes et leurs données entre les mondes britannique et français (1700-1836), Université de Paris VIII, 2017.

11. 23 juin 1759 : lettres patentes de Versailles établissant officiellement l'École de chirurgie d'Orléans. Les archives de l'école ont brûlé en 1940, mais plusieurs études locales datant du XIX ${ }^{\mathrm{e}}$ siècle (du Dr Charpignon en 1869, d'Eugène Bimbenet en 1875 et du Dr Patay en 1880) soulignent son sérieux et son importance. 
12. John V. PICKSTONE, « Les révolutions analytiques et les synthèses du modernisme », p. 33-53, in Histoire des sciences des sciences et des savoirs, Dominique PESTRE (dir.), t. 2 : Modernité et globalisation, Kapil RaJj et H. Otto SiBum (dir.), p. 40.

13. Descourtilz en rajoute un peu sur l'«intimité «: Daubenton (1716-1799) est certes directeur du nouveau Muséum d'histoire naturelle, mais il est déjà un vieillard, tout comme JacquesChristophe Valmont de Bomare (1731-1807), auteur d'un Dictionnaire raisonné universel d'histoire naturelle paru en 1768 et sans cesse réédité, en 1791 encore. Balthazar-Georges Sage (1740-1824) est quant à lui minéralogiste, fondateur de l'École des mines de Paris (Cf. Bernard DAUGERON, Collections naturalistes entre science et empires (1763-1804), Paris, Public. scientif. du MNHN, 2009, p. 273). Le naturaliste Charles n'a pu être identifié.

14. Lettre au général Dugua, SHD, GR B7 13.

15. Ibid.

16. Il épouse en 1797, à vingt ans, une héritière de Saint-Domingue, Marie-Louise Remoussin, dont la famille, liée aux Rossignol-Desdunes, possède en France le beau château de Beaumont-enGâtinais (Seine-et-Marne), situé à un kilomètre de celui de Boësses (Loiret), où se trouve le château familial des Descourtilz.

17. Les références de ce type (Voyages) renvoient aux différents tomes et pages des Voyages d'un naturaliste, op.cit., 1809.

18. Sa qualité de franc-maçon lui évite de voir sa malle fouillée mais, désormais sans moyens, il est hébergé à Cuba par un « frère » qui prend pitié de lui.

19. Roume, qui fut commissaire du gouvernement français à Tobago en 1791, a déjà une bonne connaissance du monde caraïbe et possède son propre cabinet d'histoire naturelle.

20. BERNARDIN DE SAINT-PIERRE, "Mémoire sur la nécessité de joindre une ménagerie au Jardin des Plantes de Paris ", adressé à la Convention fin 1792, Euvres complètes, Paris, Classiques Garnier, p. 346, cité par Pierre SERNA, Comme des bêtes. Histoire politique de l'animal en révolution (1750-1840), Paris, Fayard, 2017, p. 91.

21. Flore des Antilles, ou Histoire générale, botanique, rurale et économique des végétaux indigènes des Antilles, et des exotiques qu'on est parvenu à y naturaliser, Paris, chez l'auteur, 1808-1827, 4 vol. Tussac se distingue aussi par sa défense radicale des intérêts des colons, ce qui l'amène à polémiquer avec l'abbé Grégoire.

22. Il s'agit de « Haugard» (Jean Louis Hoggard), « Advénier » (Alexandre Philippe Advenier) et «Gonzalès » (Antoine Gonzalès, peintre d'origine madrilène).

23. Cf. François REGOURD, «Collecter les savoirs outre-mer: la Machine coloniale des rois de France ", in Liliane Hilaire-Pérez, Fabien Simon et Marie Thébaud-Sorger (dir.), L'Europe des sciences et des techniques. Un dialogue des savoirs, XVe-XVIII siècle, Rennes, PUR, 2016, p. 207-211. B. DAUGERON, Collections naturalistes entre science et empires (1763-1804), op. cit.

24. Jean-Luc CHAPPEY, Des naturalistes en Révolution. Les procès-verbaux de la Société d'histoire naturelle de Paris (1790-1798), Paris, CTHS, 2009, p. 42 et 65.

25. Formé auparavant par son oncle Jean-Baptiste Leblond (1747-1815), grand connaisseur des Antilles nommé médecin-botaniste du roi en 1786. Jean LESCURE, «Les voyageurs et les naturalistes français aux Antilles (XVIII ${ }^{\mathrm{e}}-\mathrm{XIX}^{\mathrm{e}}$ siècle)», p. 107-133 de L'exploration naturaliste des Antilles et de la Guyane, Actes du $123^{\mathrm{e}}$ Congrès national des sociétés historiques et scientifiques, Section Sciences, histoire des sciences et des techniques et archéologie industrielle, AntillesGuyane, 6-10 avril 1998, Jean-Loup D'HONDT et Jacqueline LORENZ (dir.), Paris, Éd. du CTHS, 2001, $237 \mathrm{p}$.

26. Ibid. Nommé directement par le comte de La Luzerne, secrétaire d'État à la marine et aux colonies, Nectoux multiplie les mémoires, dont un sur le café, culture qui vient de connaître un rapide développement. 
27. Voir Michel JANGoux (dir.), Portés par l'air du temps : les voyages du capitaine Baudin, Bruxelles, Éditions de l'Université de Bruxelles, 2010.

28. SHD, GR B7 13. Advenier (ancien élève de l'École des mines) aurait lui-même, au Port-auPrince, pour collègues, Descourtilz, Hoggard (botaniste) et Dabeneaud, et au Cap Tussac, l'abbé de la Haie et Daubertès.

29. Reproduit sous le titre «Extrait du rapport fait à l'institut de France sur un Ouvrage manuscrit relatif au Crocodile de Saint-Domingue », p. 98-104 du t. III des Voyages. Le Muséum national d'histoire naturelle conserve sous la cote Ms 598 une copie avec planches originales de ce travail premier, de la main de Descourtilz et datée de 1825 , sous le titre Histoire naturelle du crocodile à museau aigu de Antilles...

30. Louis Élie MOREAU DE SAINT-MÉRY, Description topographique, physique, civile, politique et historique de la partie française de l'isle Saint-Domingue, Paris, Dupont, 1797, t. 1, p. 24-39.

31. Cf Jean-Luc CHAPPey, La société des Observateurs de l'homme (1799-1804). Des anthropologues au temps de Bonaparte, Paris, Société des études robespierristes, 2002, 573 p.

32. Voyages, t. III, p. 109-234.

33. Histoire naturelle du genre humain de l'an IX. Voir Claude BLANCKAERT, « 1800 - Le moment «naturaliste» des sciences de l'homme», Revue d'Histoire des Sciences Humaines, 2000/2 (no 3), p. 117-160.

34. Une copie in folio de son traité sur le caïman, de la main de Descourtilz, se trouve au Muséum national d'histoire naturelle sous la cote Ms 598. Datée de 1825, elle est illustrée de gouaches peintes par son fils Jean-Théodore.

35. Service historique de la Défense, Vincennes, Armée de St Domingue, Correspondance 1799-1812, ms B7 13. Transcription : M. C. Kiener.

36. Texte reproduit in extenso dans Les instructions scientifiques pour les voyageurs (XVIIe-XIXe siècles) , textes choisis et présentés par Silvia CollinI, Antonia VANNONI (dir.), Paris, L'Harmattan, 2005, p. 93-100.

37. Ibid., p. 93-94

38. Voir notamment : Philippe R. GIRARD, Ces esclaves qui ont vaincu Napoléon. Toussaint Louverture et la guerre d'indépendance haïtienne, Bécherel, Les Perséides, 2013 ; Yves BENOT et Marcel DORIGNY (dir.), Rétablissement de l'esclavage dans les colonies françaises, 1802. Ruptures et continuité de la politique coloniale française (1800-1830). Aux origines d'Haïti, Paris, Maisonneuve \& Larose, 2003.

39. Philippe R. GIRARD, Ces esclaves, ..., op.cit., p. 179-181 sur le cessez-le-feu et p. 208-209 sur son arrestation.

40. Il en tirera le témoignage inséré dans ses Voyages, dont on trouvera l'édition critique dans Vincent COUSSEAU \& Michel C. KIENER, La Révolution à Saint-Domingue..., op. cit., p. 88-178.

41. Jean-Luc CHAPPEY, "Utopies en contexte. Questions sur le statut du pédagogue sous le Directoire » p. 5, La Révolution française. Cahiers de l'Institut d'histoire de la Révolution française, $\mathrm{n}^{\circ} 4$ (2013), Pédagogies, utopies et révolutions (1789-1848). https://journals.openedition.org/lrf/791. Consulté en août 2018.

42. Pietro CORSI, «Histoire naturelle continuité et rupture, 1795-1805», Communication au colloque Rénovation et mutations des savoirs, 1795-1805, Paris (Centre culturel italien), 15-16 octobre 1999.

43. Jean-Luc CHAPPEY, Des naturalistes en Révolution, op.cit., p. 38-57.

44. Pierre-Yves LACour, La République naturaliste. Collections d'histoire naturelle. Révolution française (1784-1804), Paris, Public. scientif. du MNHN, 2014.

45. Détail des questions dans Les instructions scientifiques pour les voyageurs..., op. cit., p. 96-100.

46. Le capitaine devait veiller à ce qu'aucune verroterie ne soit embarquée à bord. On trouve sous la cote unique ms 2621 copie des instructions pour le Citoyen Baudin, capitaine de la flûte la Belle Angélique, signée par le ministre de la marine et des colonies Bréguet, données à Paris le 26 
fructidor an $4^{\mathrm{e}}$ (12 sept 1796), et les Instructions données par le Museum d'histoire naturelle aux Naturalistes qui accompagnent le C. Baudin dans son expédition à l'Isle de la Trinité Espagnole, document de treize pages adressé « au $C^{\text {en }}$ Larevellière-Lepeaux », et signé "Jussieu directeur du museum d'histoire naturelle », 28 fructidor An 4 (14 sept 1796).

47. Paris, A. Belin imprimeur, 1818, 47 pages.

48. Michael S. OSBORNE, « Applied Natural History and Utilitarian Ideals: "Jacobin Science" at the Museum d'Histoire Naturelle, 1789-1870 ", in Bryant T. RAGAN et Elizabeth A. WILLIAMS (dir.) Recreating Authority in Revolutionary France, New-Brunswick, Rutgers University Press, 1992, p. 125-210.

49. Voir à ce propos Pierre SERNA, Comme des bêtes..., op.cit., p. 90-96 et p. 149-214.

50. Ibid., p. 96.

51. Observations... [octobre 1793], Musée national d'histoire naturelle, ms 299, cité par Lorelai KURY, Histoire naturelle et voyages scientifiques (1780-1830), Paris, L'Harmattan, 2001, p. 35.

52. Jean-Luc CHAPPEY, Des naturalistes en révolution, op. cit., p. 201-205.

53. Ibid., p. 207.

54. Consulter à ce propos la thèse de Lorelai KURY, Civiliser la nature: histoire naturelle et voyages (France, fin du XVIII ${ }^{e}$ siècle-début du XIX ${ }^{e}$ siècle), EHESS, 1995.

55. Ibidem.

56. François REGOURD, «Collecter les savoirs outre-mer... ", article cité ; James E. MCCLELLAN et François REgourd, The Colonial Machine: French Science and Overseas Expansion in the Old Regime, Turnhout, Brepols, 2011.

57. Service Historique de la Défense, Armée de terre, B7/26.

58. Il aboutit après un «travail de vingt années, obtenu par des sacrifices énormes " à une Flore médicale des Antilles ou Traité des plantes usuelles... peinte d'après les dessins faits sur les lieux par M. J. Th. Descourtilz (Pichard, 8 vol., 1821-1829), dont un exemplaire portant le cachet de Cuvier est conservé à la bibliothèque du Muséum. "Ouvrage arraché miraculeusement à l'incendie de St Domingue [...], cette flore m'est d'autant plus chère, écrit Descourtilz au duc de Richelieu, qu'elle m'a sauvé la vie au milieu du massacre des blancs par les nègres révoltés. » (Bibliothèque de la Sorbonne, manuscrits de la Bibliothèque Richelieu 106, lettre de Descourtilz au duc de Richelieu, ministre de l'Intérieur, 9 octobre 1821).

\section{RÉSUMÉS}

Après avoir séjourné trois années à Saint-Domingue, le jeune et méconnu naturaliste Michel Étienne Descourtilz soumet en 1802 aux autorités son Projet d'organisation d'un Lycée colonial. Cette nouvelle institution avait pour objectif de donner un nouvel élan, dans la colonie elle-même, à une activité scientifique en berne du fait des troubles. Le plan, qui restera sans suite, est marqué du sceau de l'utilitarisme et de l'exploitation coloniale. Toutefois, il traduit aussi une démarche scientifique novatrice, fondée sur l'observation du vivant dans son milieu et émancipée du centralisme scientifique de la capitale.

After spending three years in Saint-Domingue, the young and unknown naturalist Michel Étienne Descourtilz submitted in 1802 to the authorities his project to establish a Lycée colonial. This new institution was intended to give a new impulse, in the colony itself, to a scientific activity that 
had been weakened by the war and revolts. The plan, which will remain unfulfilled, is marked by utilitarianism and colonial exploitation. However, it also reflects an innovative scientific approach, based on the observation of life in its environment and emancipated from the scientific centralism of the capital.

INDEX

Keywords : Descourtilz Michel-Étienne, natural sciences, naturalists, Saint-Domingue, Haiti, Tussac François-Richard de

Mots-clés : Descourtilz Michel-Étienne, sciences naturelles, naturalistes, Saint-Domingue, Haïti, Tussac François-Richard de

\section{AUTEURS}

\section{VINCENT COUSSEAU}

Université de Limoges

MICHEL C. KIENER

Professeur à la retraite

Université de Limoges 